\title{
Role of Ultrasonography in the Diagnosis of Myocysticercosis
}

\author{
Mohini Chaudhary ${ }^{1}$, Deepak Singla ${ }^{1}$, Shruti Chandak ${ }^{2}$, Ashutosh Kumar ${ }^{3}$, Arjit Agarwal ${ }^{4}$, Vijai Pratap ${ }^{2}$ \\ ${ }^{1}$ Junior Resident, Department of Radio-Diagnosis, Teerthanker Mahaveer Medical College And Research Center (TMMC\&RC), Moradabad, ${ }^{2}$ Professor, Department \\ of Radio-Diagnosis, Teerthanker Mahaveer Medical College And Research Center (TMMC\&RC), Moradabad, ${ }^{3}$ Professor, Department of Pathology, Teerthanker \\ Mahaveer Medical College And Research Center (TMMC\&RC), Moradabad. ${ }^{4}$ Associate Professor, Department of Radio-Diagnosis, Teerthanker Mahaveer Medical \\ College And Research Center (TMMC\&RC), Moradabad.
}

\section{Abstract}

Background: Myocysticercosis is a parasitic infestation caused by encysted larvae of taenia solium. The infection occurs due to ingestion of taenia eggs in raw or undercooked pork, contaminated water or vegetables which are disseminated by hematogenous route to various tissues of the human body. Myocysticercosis occurs due to muscular involvement by the parasite and is more common in population having poor access to sanitation facilities. Ultrasonography is a convenient, easily accessible and cost effective modality which can be used to demonstrate the various appearances of myocysticercosis. FNAC is diagnostic and helpful in identifying the parasite and its body parts. Aim and Objective: To evaluate the role of ultrasonogaphy in the diagnosis of myocysticercosis. Subjects and Methods: The study was conducted in the Radiology department of Teerthanker Mahaveer Medical College and Research Centre, Moradabad. We included 50 pathologically confirmed cases of myocysticercosis who had undergone ultrasound evaluation by linear ultrasound probe 9L4 of Siemens Acuson S2000 ultrasound machine Results: Out of 50 patients that were enrolled in our study, the most common appearance was of well defined cystic lesion of variable size with an eccentric, echogenic scolex. Other appearances include ill defined cystic lesion with or without scolex or a completely calcified myocysticercosis. Most common location was found to be anterior abdominal wall followed by lower extremities, upper extremities and facial muscles subsequently. Conclusion: We concluded that ultrasound is an indispensable tool for the diagnosis of mycocysticercosis with characteristic diagnostic appearances.

Keywords: Myocysticercosis, Ultrasonography.

Corresponding Author: Dr. Deepak Singla, Junior Resident, Department of Radio-Diagnosis, Teerthanker Mahaveer Medical College And Research Center (TMMC\&RC), Moradabad.

Received: February 2019

Accepted: February 2019

\section{Introduction}

Cysticercosis is a parasitic infestation caused by encysted larvae of Taenia solium that is mainly found in pigs. Pigs serve as intermediate hosts whereas human beings serve as either definitive or an intermediate host for adult tapeworm. ${ }^{[1]}$

The infection occurs due to ingestion of Taenia eggs in raw or undercooked pork, contaminated water or vegetables. ${ }^{[2]}$ The larvae are then disseminated by hematogenous route to various tissues of the human body. Cysticercosis is more common in countries having poor access to sanitation facilities. ${ }^{[3]}$ Approximately $86 \%$ of the cases of cysticercosis are ocular and cerebral in location and rest are found in subcutaneous, cardiac, pulmonary, muscular, hepatic and oral locations. ${ }^{[4]}$

Ultrasonography is a convenient, easily accessible and cost effective modality which can be used to demonstrate the various appearances of myocysticercosis in its different stages of evolution which further aids in diagnosis.

The diagnosis can be confirmed by cytopathology or histopathology which shows parasitic body parts which have undulating cuticular layer and inner nucleus and

\section{$\underline{\text { AIM }}$}

To evaluate the role of ultrasonography in the diagnosis of myocysticercosis.

\section{Subjects and Methods}

The study was conducted in the Radiology department of Teerthanker Mahaveer Medical College and Research Centre, Moradabad. We included 50 confirmed cases of myocysticercosis who had undergone ultrasound evaluation by linear ultrasound probe 9L4 of Siemens Acuson S2000 ultrasound machine.

\section{Results}

\section{Age Group:}

Out of 50 patients that were enrolled in our study the majority of the cases (20) having myocysticercosis were in the age group of $21-30$ years. 11 cases were in $11-20$ years of age group.

Sex: 
Chaudhary et al; Ultrasanagraphy in the Diagnasis of Myacysticercasis

Total no. of males having myocysticercosis were 27 and rest were females in our study.

\begin{tabular}{|c|c|c|c|c|c|}
\hline Age & $\begin{array}{c}\text { Total } \\
\text { Cases }\end{array}$ & \multicolumn{2}{|c|}{ Male No. Percentage } & \multicolumn{2}{c|}{$\begin{array}{c}\text { Female No. } \\
\text { Percentage }\end{array}$} \\
\hline $0-10$ & 4 & 2 & $50 \%$ & 2 & $50 \%$ \\
\hline $11-20$ & 11 & 6 & $54.5 \%$ & 5 & $45.5 \%$ \\
\hline $21-30$ & 20 & 12 & $60 \%$ & 8 & $40 \%$ \\
\hline $31-40$ & 8 & 3 & $37.5 \%$ & 5 & $62.5 \%$ \\
\hline$>40$ & 7 & 4 & $57.2 \%$ & 3 & $42.8 \%$ \\
\hline
\end{tabular}

\section{Appearances on Ultrasonography:}

We found various sonographic appearances of myocysticercosis in our study. The most common appearance was of well defined cystic lesion of variable size with an eccentric, echogenic scolex inside. Other appearances included ill defined cystic lesion with or without scolex or a completely calcified myocysticercosis. In some cases perilesional oedema was also noted.

\begin{tabular}{|c|c|}
\hline Appearance & No. of cases \\
\hline Well defined cystic lesion with eccentric scolex & 29 \\
\hline Ill defined cystic lesion with or without scolex & 7 \\
\hline Associated perilesional edema & 6 \\
\hline Calcified myocysticercosis & 8 \\
\hline
\end{tabular}

\section{$\underline{\text { Site }}$}

The most common sites for myocysticercosis noted in our study was anterior abdominal wall followed by lower extremities, upper extremities and facial muscles.

\begin{tabular}{|c|c|}
\hline Site & No. Of cases \\
\hline Anterior abdominal wall & 18 \\
\hline Lower extremities & 12 \\
\hline Upper extremities & 10 \\
\hline Facial muscles & 7 \\
\hline Others & 3 \\
\hline
\end{tabular}

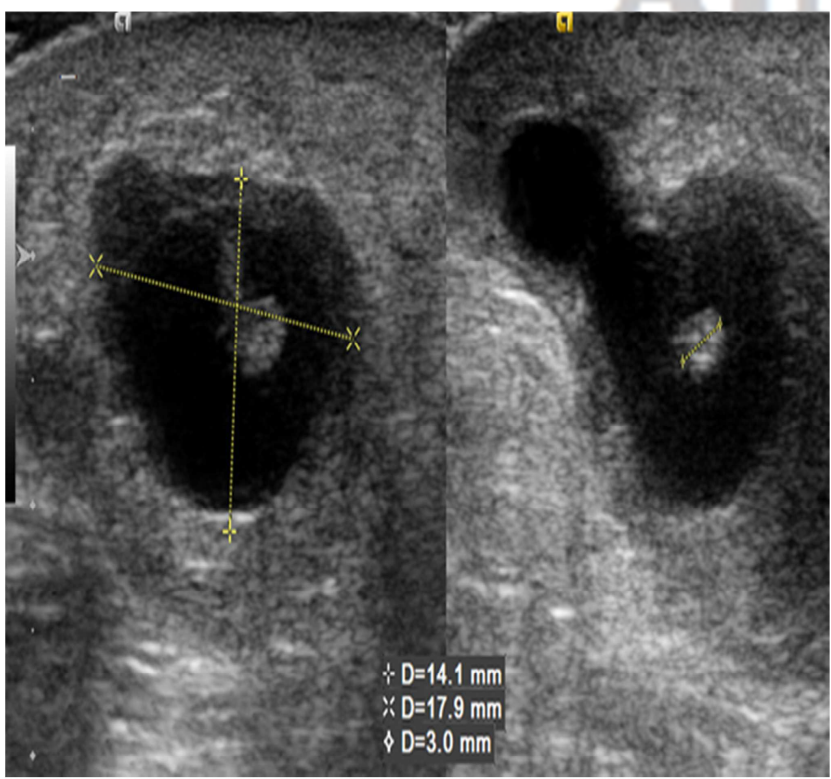

Figure 1: Greyscale ultrasound images showing the presence of well defined anechoic cystic lesion with eccentric echogenic scolex with mild perilesional edema.

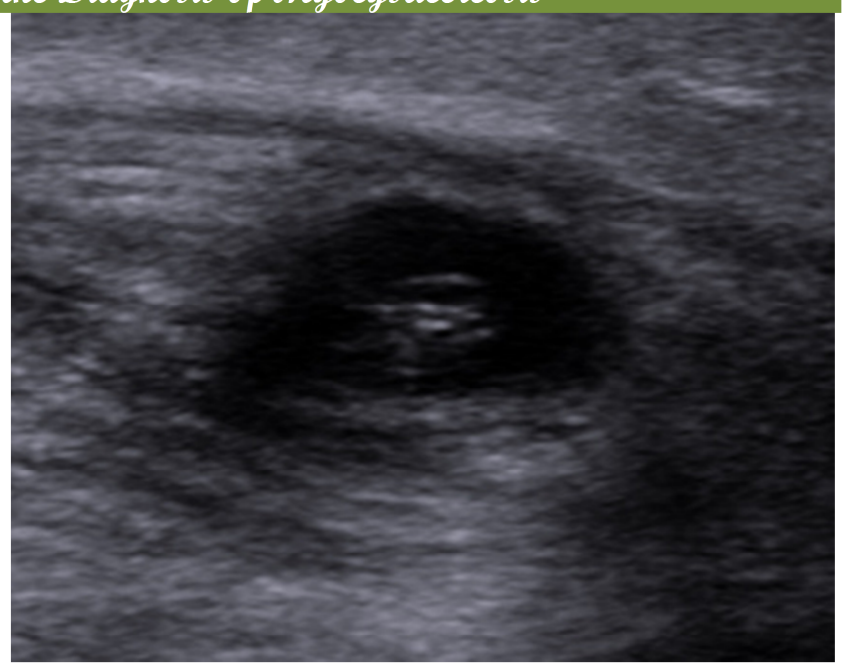

Figure 2: Grey scale ultrasound image showing a well defined, anechoic cystic lesion with internal echogenic content.

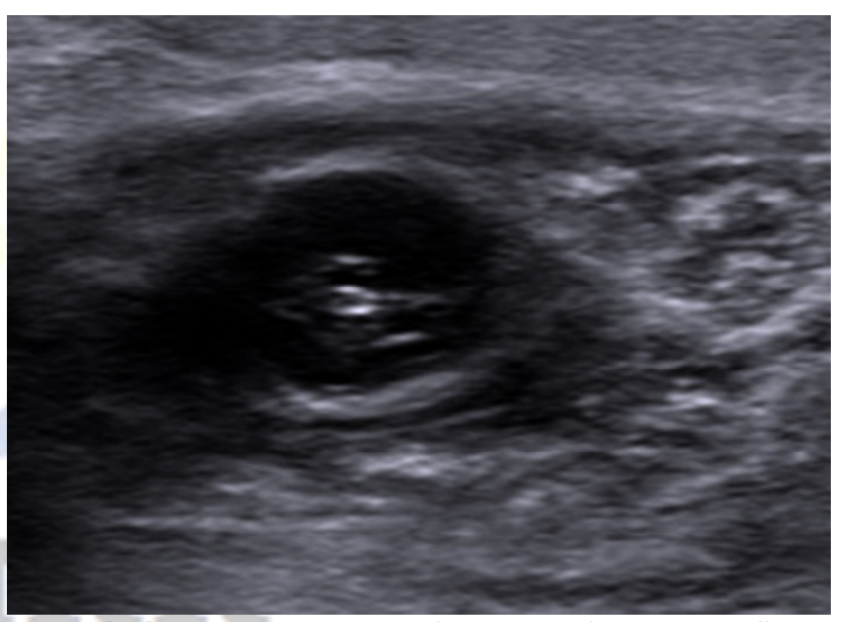

Figure 3: Grey scale ultrasound image showing a well defined, anechoic cystic lesion with perilesional edema and fluid collection.

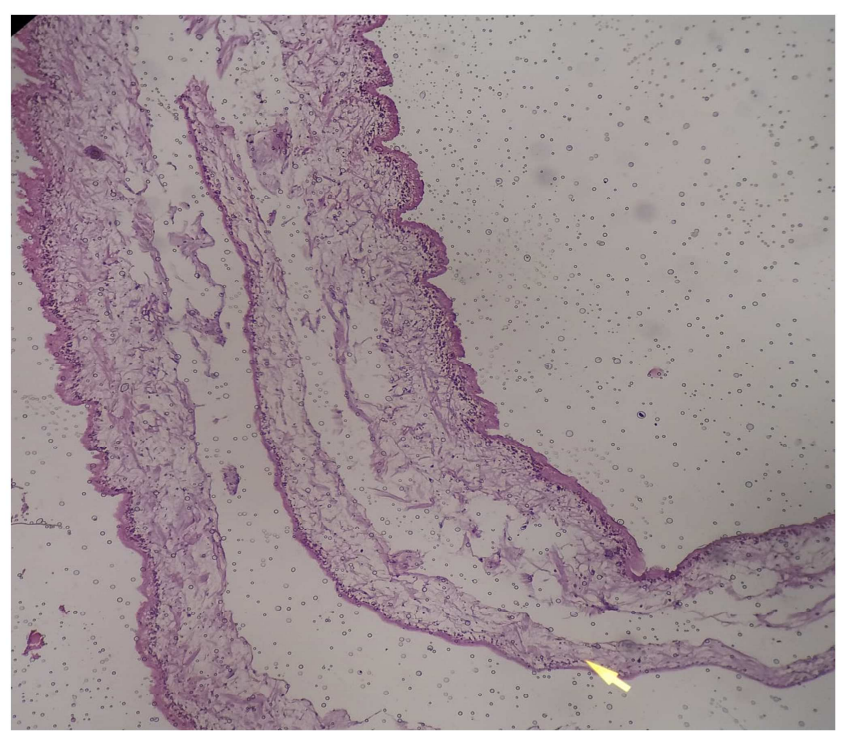

Figure 4: parasitic body parts which have undulating cuticular layer and inner nucleus and myxoid layer. 


\section{Discussion}

Myocysticercosis in humans is caused by the larval form (cysticercus cellulosae) of the Taenia solium. It is transmitted to humans by ingestion of eggs from contaminated water, food or by regurgitation of eggs into the stomach in people infected with adult tapeworm. The eggs are hatched releasing oncospheres in the small bowel which penetrates the mucosa of the small bowel to reach various organs through hematogenous route, where they develop to larval form. These eggs are excreted in the human fecal matter, which are then ingested by the pig. On ingestion, the eggs get hatched in the small bowcl of the pig leading to cysticercosis thus completing the cycle. However, humans can accidentally be intermediate hosts, manifesting cysticercosis. They remain viable in the larval stage for many years and evades immune recognition. Granulomatous inflammatory response occurs following the death of larva producing various symptoms, depending upon anatomical location. ${ }^{[5]}$

"In the muscular form, three distinct types of clinical manifestations have been described":

- Myalgic type

- Mass like or Pseudotumor or Abscess like type

- Pseudohypertrophic type, ${ }^{[6,7]}$

Death of the larva results in leakage of fluid from the cyst leading to inflammation causing local pain and myalgia as noted in most of the patients. Secondly, there can be intermittent leakage of fluid due to degeneration of the cyst, leading to chronic inflammation and pericystic collection, known as the mass like, pseudotumor, or abscess like type. Alternatively, retraction of cyst with thickening of capsule and calcification of scolex can be seen. In the end complete calcification of the cyst occurs.

Cysticercosis commonly involves the brain and orbit but can also be seen in subcutaneous and muscular forms. The latter two forms are usually asymptomatic. ${ }^{[8,9]}$

On Contrast Enhanced Computed Tomography or Contrast Enhanced Magnetic Resonance Imaging, Neurocysticercosis appears as well defined, ring enhancing lesion with eccentric nodule and can also give characteristic "starry sky" appearance.

We found various sonographic appearances of myocysticercosis in our study but the most common appearance was of well defined, small, elliptical cystic lesion with an eccentric, echogenic scolex inside [Figure 1]. Other appearances include ill defined cystic lesion with or without scolex [Figure 2] or a completely calcified myocysticercosis. In some cases perilesional oedema with collection was also noted [Figure 3].

The most common sites for myocysticercosis noted in our study was anterior abdominal wall followed by lower extremities, upper extremities and facial muscles subsequently.

Confirmation of diagnosis is done by cytopathological or histopathological evaluation which shows undulating cuticular layer, inner nucleus and myxoid layer of parasite ¡Figure 4ך.

\section{Conclusion}

The ultrasound is an indispensable tool for the diagnosis of myocysticercosis with characteristic diagnostic appearances.

References

1. Arora P, Devi P, Wazir S et al. Importance of ultrasonography and magnetic resonance imaging in diagnosis of cysticercosis of temporalis muscle mimicking temporal space infection. Contem Clin Dent. 2013;4(4):504-8.

2. Ribeiro AC, Luvizotto MC, Soubhia AM, de Castro AL. Oral cysticercosis: case report. Oral Surg Oral Med, Oral Pathol Oral Radiol Endod. 2007;104(4):e56-8.

3. Ramakrishnan P, Bahirwani S, Balaji P. Cysticercosis of the masseter. Indian J Dent Res. 2012;23(3):436-8.

4. Mahajan D, Khurana N, Setia N. Coexistence of salivary gland cysticercosis with squamous cell carcinoma of the mandible. Oral Surg Oral Med, Oral Pathol Oral Radiol Endod. 2007;103(3):e47-50.

5. Vijayaraghavan SB. Sonographic appearances in cysticercosis. J Ultrasound Med. 2004;23:423-7.

6. Anderson GA, Chandi SM. Cysticercosis of the flexor digitorum profundus muscle producing flexion deformity of the fingers. J Hand Surg. 1993;18(3):360-2.

7. Chopra JS, Nand N, Jain K, Mittal R, Abrol L. Generalized muscular pseudohypertrophy in cysticercosis. Postgrad Med J. 1986;62:299300 .

8. Schmidt DKT, Jordaan HF, Schneider JW. Cerebral and subcutaneous cysticercosis treated with albendazole. Int J Dermatol. 1995:34:574-9

9. Yamashita P, Kelsey J, Henderson SO. Subcutaneous cysticercosis. J Emerg Med. 1998;16:583-6.

Copyright: (C) the author(s), publisher. Asian Journal of Medical Radiological Research is an Official Publication of "Society for Health Care \& Research Development”. It is an open-access article distributed under the terms of the Creative Commons Attribution Non-Commercial License, which permits unrestricted non-commercial use, distribution, and reproduction in any medium, provided the original work is properly cited.

How to cite this article: Chaudhary M, Singla D, Chandak S, Kumar A, Agarwal A, Pratap V. Role of Ultrasonography in the Diagnosis of Myocysticercosis. Asian J. Med. Radiol. Res. 2019;7(1):26-28.

DOI: dx.doi.org/10.21276/ajmrr.2019.7.1.6

Source of Support: Nil, Conflict of Interest: None declared. 